\title{
First Two Brazilian Cases: Correction of Ascending Aortic Aneurysm and Aortic Valve Stenosis with Sutureless/Rapid Deployment Aortic Prosthesis
}

Isaac Azevedo Silva'1, MD, PhD; Ricardo Barros Corso', MD; Marcus Vinicius Nascimento Santos ${ }^{1}$, MD; Helmgton José Brito de Souza', MD, PhD; Glauco K. S. Pina ${ }^{1}$, MD

DOI: 10.21470/1678-9741-2018-0148

\begin{abstract}
Ascending aortic aneurysm is usually associated with aortic valve diseases, especially aortic stenosis. The standard technique involves the substitution of the dilated aortic segment with a Dacron tube and replacement of the aortic valve with a regular prosthesis. The correction of ascending aortic aneurysm with
\end{abstract}

aortic valve replacement using the new sutureless and rapid deployment prosthesis was performed by a minimally invasive approach and for the first time in a Brazilian Center.

Keywords: Aortic Valve - Surgery. Heart Valve Prosthesis. Treatment Outcome.

\section{INTRODUCTION}

The association between aortic valve dysfunction and dilatation of the ascending aorta is well known ${ }^{[1]}$ and the treatment usually involves the aortic valve replacement with substitution of the dilated aortic segment. Sometimes, a total ascending aortic segment needs to be treated in addiction of a valve replacement, (Bentall De Bono) but an aortic-root-sparing approach may be feasible when there is no sinus dilatation ${ }^{[2]}$ In this new era of sutureless and rapid deployment prosthesis, a root-sparing ascending aortic replacement with modern prosthesis has been described with good results ${ }^{[3]}$. Here, we describe our initial two cases, for the first time performed in Brazil.

\section{CASE REPORT}

\section{Case 1}

A 62-year-old male patient, with no history of comorbidities, presented with functional capacity reduction and complained of dyspnea on greater efforts of progressive worsening. Coronary angiography showed no obstructive lesions in coronary arteries.

\section{'Cardiovascular Associados, Brasilia, DF, Brazil.}

This study was carried out at the Cardiovascular Associados, Brasilia, DF, Brazil.
Echocardiogram: EF 73\%; Left atrium $37 \mathrm{ml} / \mathrm{m}^{2}$; Aortic valve area $1.0 \mathrm{~cm}^{2}$; maximum gradient $81 \mathrm{mmHg}$ and medium gradient $51 \mathrm{mmHg}$; Pulmonary artery systolic pressure $33 \mathrm{mmHg}$; mitral valve without abnormalities.

Angiotomography: root $35 \mathrm{~mm}$; tubular thread ascending $47 \mathrm{~mm}$; arch $42 \mathrm{~mm}$; isthmus $25 \mathrm{~mm}$; descending $22 \mathrm{~mm}$.

He was submitted to an aortic valve replacement + replacement of the ascending aorta by the minimally invasive technique (mini-sternotomy in J). Briefly, under cardiopulmonary bypass, the distal ascending aorta was cross clamped. After anterior oblique aortotomy, a calcified and stenotic tricuspid aortic valve was found. Del Nido cardioplegia was delivered directly in coronary ostia. Resection of the entire dilated aortic segment was performed and a Dacron ${ }^{\mathrm{TM}}$ tube number 34 was sutured above the coronary ostia and a biological sutureless aortic valve (Perceval ${ }^{\mathrm{TM}}$ XL LivaNova) was implanted. Cross clamp time: $81 \mathrm{~min}$. Total cardiopulmonary bypass time was $95 \mathrm{~min}$.

Intraoperative transesophageal echocardiogram demonstrated normofunction of the valvular prosthesis, no paravalvular leak and a mean gradient of $3 \mathrm{mmHg}$.

Correspondence Address:

Isaac Azevedo Silva

iD https://orcid.org/0000-0003-1869-6272

Cardiovascular Associados

Seps 709/909 - Bloco A, Sala T04 - Brasilia, DF, Brazil

Zip Code: 70390-095

E-mail: isaacazevedo@yahoo.com 


\section{Case 2}

A 61-year-old male patient, with a history of hypertension and a long-term heart murmur accompanied of recent onset of dizziness and dyspnea. Echocardiogram showed severe aortic stenosis and ascending aortic aneurysm. Angiotomography showed ascending aorta with $48 \mathrm{~mm}$ diameter. Coronary cardiac catheterization with irregularities and severe aortic stenosis, with a systolic gradient of $80 \mathrm{mmHg}$.

He was submitted to aortic valve replacement + replacement of the ascending aorta by the minimally invasive technique (ministernotomy in J). Under cardiopulmonary bypass an anterior oblique aortotomy was performed. A type 1 bicuspid aortic valve was found, full of calcium and very stenotic. Del Nido cardioplegia was delivered directly in coronary ostia. Resection of the entire dilated aortic segment was performed and a Dacron tube number 32 was sutured above the coronary ostia and a biological rapid deployment valve (Intuity ${ }^{\text {тм }} 25$ Edwards) was implanted. Cross clamp time: 80 min. Total cardiopulmonary bypass time was 96 min.

Intraoperative transesophageal echocardiogram demonstrated preserved biventricular function, well positioned prosthesis, no paravalvular leak, mean gradient of $4 \mathrm{mmHg}$.

Both patients had an uneventful recovery and are doing well.

\section{DISCUSSION}

Aortic surgeries with aortic valve replacement are commonly time-consuming procedures. The use of available sutureless and rapid deployment valves have resulted in an important cross-clamp and by-pass time reduction ${ }^{[4]}$ in comparison with regular valves, at least in valve replacement procedures. Lio et al. ${ }^{[3]}$ reported successful utilization of non-conventional valves in combined surgeries (aorta and aortic valve) with reasonable time sparing. Considering the full regulatory acceptance, the growing utilization of these valves around the world and, especially because of their comfortable implantation, complex procedures can be performed in a simpler way. In our cases, the minimally invasive technic was applied, cross-clamp and by-pass time were reasonable, and the results were very good.

\section{No financial support. \\ No conflict of interest.}

\begin{tabular}{ll}
\hline Authors' roles \& responsibilities \\
\hline IAS & $\begin{array}{l}\text { Wrote the paper; final approval of the version to be } \\
\text { published } \\
\text { Reviewed and performed critical analysis; final approval } \\
\text { of the version to be published }\end{array}$ \\
MBC $\quad \begin{array}{l}\text { Reviewed and performed critical analysis; final approval } \\
\text { of the version to be published }\end{array}$ \\
HJBS $\quad \begin{array}{l}\text { Reviewed and performed critical analysis; final approval } \\
\text { of the version to be published } \\
\text { Reviewed and performed critical analysis; final approval } \\
\text { of the version to be published }\end{array}$ \\
GKSP
\end{tabular}

\section{REFERENCES}

1. Cotrufo M, Agozzino L, De Feo M, Della Corte A, De Santo LS, et al. Aortic valve dyfunction and dilated ascending aorta. A complex and controversial association. Ital Heart J [Internet]. 2003 [cited 2019 Apr 30];4(9):589-95. Available from: http://www.federcardio.it/pdf/2003/09/20030206.pdf.

2. Peterss S, Charilaou P, Dumfarth J, Li Y, Bhandari R, Tranquilli M, et al. Aortic valve disease with ascending aortic aneurysm: impact of concomitant rootsparing (supracoronary) aortic replacement in nonsyndromic patients. J Thorac Cardiovasc Surg. 2016;152(3):791-798. doi:10.1016/j.jtcvs.2016.05.020.

3. Lio A, Ferrarini M, Miceli A, Glauber M. Sutureless prosthesis implantation and ascending aorta replacement through a ministernotomy approach. Innovations (Phila). 2018;13(3):239-41. doi:10.1097/IMI.0000000000000479.

4. Miceli A, Santarpino G, Pfeiffer S, Murzi M, Gilmanov D, Concistré G, et al. Minimally invasive aortic valve replacement with perceval $S$ sutureless valve: early outcomes and one-year survival from two European centers. J Thorac Cardiovasc Surg. 2014;148(6):2838-43. doi:10.1016/j.jtcvs.2014.02.085. 